\title{
Correspondence
}

Korean J Ophthalmol 2021;35(3):::242-243

https://doi.org/10.3341/kjo.2021.0002

\section{Use of Nd: YAG Laser to Recanalize XEN Gel Stent Occluded by Iris Pig- ment}

\section{Dear Editor,}

The XEN gel stent (Allergan Plc, Dublin, Ireland), developed using biocompatible hydrophilic material, has been spotlighted as a device that can be surgically implanted to effectively control intraocular pressure (IOP) with fewer and less severe postoperative complications compared with conventional glaucoma filtering surgery [1]. However, within a week of XEN gel stent implantation, about $4 \%$ of patients experience early-postoperative lumen blockage by blood clot, iris pigment or fibrin [2]. Also, late obstruction can be caused by chronic intraocular inflammation [3]. We describe herein a case of late-postoperative XEN gel stent obstruction by iris pigment that was resolved using the neodymium-doped yttrium aluminum garnet (Nd: YAG) laser.

A 54-year-old male underwent XEN gel stent implantation in his left eye for pigmentary glaucoma. Previously the patient had undergone pars plana vitrectomy and trans-scleral fixation of intraocular lens due to intraocular lens dislocation in both eyes. Subsequently, reverse pupillary block occurred in the left eye, and the patient underwent laser peripheral iridotomy. Despite the maximum tolerated medical therapy and selective laser trabeculoplasty, the IOP in the left eye was uncontrolled at $27 \mathrm{mmHg}$.

Implantation of the XEN gel stent was performed in a routine fashion without any intraoperative complications. The day after surgery, the IOP was $2 \mathrm{mmHg}$, and on slit-

Received: January 6, 2021 Final revision: April 1, 2021

Accepted: April 15, 2021 lamp and gonioscopic examinations, the tip of the XEN gel stent was well positioned at the anterior trabecular meshwork level with the $1+$ cell in the anterior chamber (AC). The IOP had gradually normalized to $12 \mathrm{mmHg}$ by postoperative 2 weeks. At 2 months postoperatively, the IOP had increased to $20 \mathrm{mmHg}$, so bleb needling was performed. After that, the IOP decreased to $12 \mathrm{mmHg}$.

Five months postoperatively, the IOP had increased again to $22 \mathrm{mmHg}$, and did not respond to digital ocular compression. The $3+$ cell was seen in the AC, which was mostly iris pigment, and on slit-lamp and anterior segment optical coherence tomography, lumen blockage of the XEN gel stent at its proximal (AC side) opening was suspected (Fig. 1A-1C). AC irrigation and prophylactic bleb needling with subconjunctival 5-fluorouracil injection $(5 \mathrm{mg})$ was performed, but failed to reduce the IOP. Four shots (total energy $2.2 \mathrm{~mJ}$ ) of Nd: YAG laser ablation to the proximal end were performed to reopen the lumen, and resultantly, the IOP decreased to $10 \mathrm{mmHg}$ (Fig. 1D-1F), and remained stable 5 months afterwards.

There are several previous reports of XEN gel stent lumen blockage. Eagle and Razeghinejad reported proximal-end obstruction by iris pigment, similarly to the current report, but theirs was an instance of early-postoperative obstruction (7 days) in a primary open-angle glaucoma patient for whom the stent was removed surgically [4]. Scantling-Birch et al [2]. reported lumen blockage that was cleared by Nd: YAG laser ablation. According to that report, the lumen was obstructed by fibrin on the day after XEN gel stent implantation combined with phacoemulsification and goniosynechiolysis for primary angle-closure glaucoma. There is also another report, this one of proximal end-lumen blockage by fibrin after XEN-augmented Baerveldt surgery for secondary glaucoma. This blockage, which had occurred 1 month postoperatively, was initially treated by failed YAG-laser fibrinolysis, and subsequently by successful replacement of the stent by a thicker-lumened Baerveldt tube [5]. 

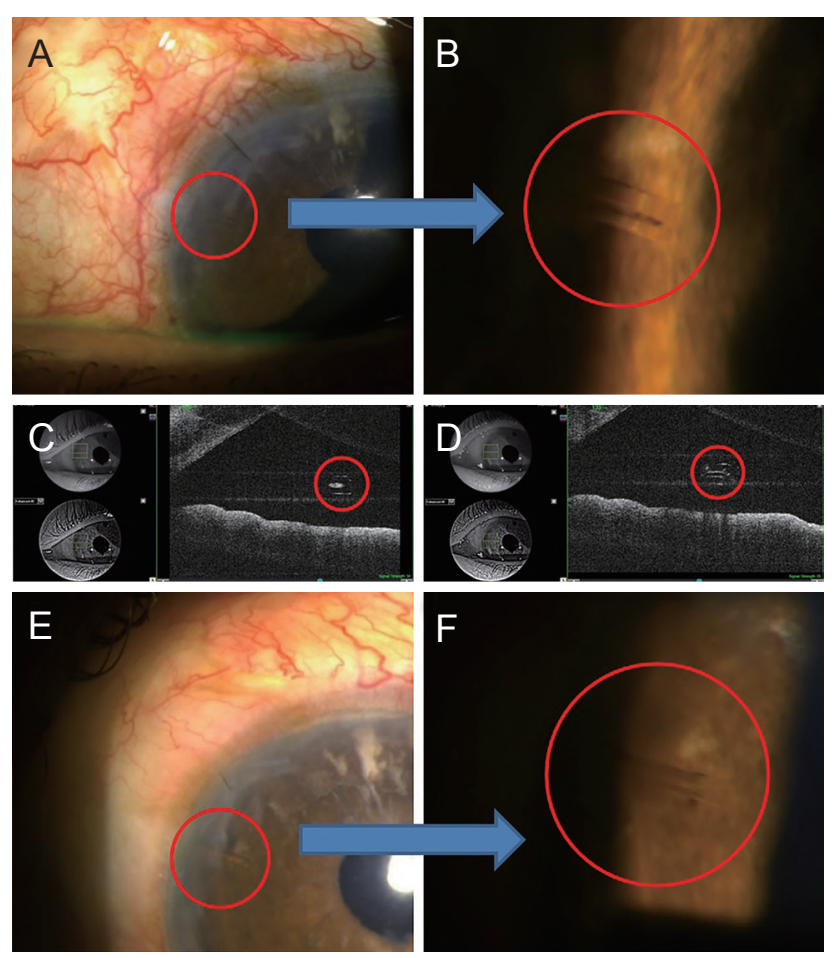

Fig. 1. (A,B) Slit-lamp exam appearance before neodymium-doped yttrium aluminum garnet (Nd: YAG) ablation, showing blockage of XEN gel stent lumen by iris pigment (red circle). (C) Anterior segment optical coherence tomography before Nd: YAG ablation confirming blockage of lumen (red circle) by hyper-reflective iris pigment. (D) Anterior segment optical coherence tomography finding (red circle) after Nd: YAG ablation showing recanalization of lumen. (E,F) Slit-lamp exam appearance (red circle).

What differentiates the current case from the relevant previous reports are the facts that the lumen blockage of the XEN gel stent occurred 5 months postoperatively by iris pigment after recurred pigment storm in a pigmentary glaucoma patient and that the obstruction was successfully treated with an Nd: YAG laser.

What should be highlighted in this case is the fact that the Nd: YAG laser can be used to resolve lumen blockage by iris pigment after XEN gel stent implantation. Although there is a case wherein a fibrin-blocked lumen after XEN gel stent implantation was reopened with the Nd: YAG laser [2], there are as yet no reports of Nd: YAG laser reopening of lumens blocked with iris pigment. Using less-invasive Nd: YAG laser ablation to recanalize the obstructed lumen could reduce the potential risk of surgical complication while preserving the XEN gel stent.

In conclusion, after XEN gel stent implantation, delayed lumen blockage by iris pigment may occur, but the lumen can be opened through Nd: YAG laser ablation.

\section{Jae Gon Kim, Chong Eun Lee}

Department of Ophthalmology, Keimyung University School of Medicine, Daegu, Korea

E-mail (Chong Eun Lee): celee@dsmc.or.kr

\section{Conflict of Interest}

No potential conflict of interest relevant to this article was reported.

\section{References}

1. Do AT, Parikh H, Panarelli JF. Subconjunctival microinvasive glaucoma surgeries: an update on the Xen gel stent and the PreserFlo MicroShunt. Curr Opin Ophthalmol 2020; 31:132-8.

2. Scantling-Birch Y, Merzougui W, Lindfield D. Early postoperative lumen blockage of ab-interno gel stent (XEN) cleared with Nd:YAG laser. Indian J Ophthalmol 2020; 68:524.

3. Gillmann K, Mansouri K, Bravetti GE, Mermoud A. Chronic intraocular inflammation as a risk factor for XEN gel stent occlusion: a case of microscopic examination of a fibrin-obstructed XEN stent. J Glaucoma 2018;27:739-41.

4. Eagle RC Jr, Razeghinejad R. Xen gel stent occlusion with iris pigment epithelium. Clin Exp Ophthalmol 2020;48:2589.

5. Gillmann K, Bravetti GE, Mansouri K. Delayed obstruction of XEN gel stent by cell debris in primary open-angle glaucoma: a new insight into the pathophysiology of filtration device failure. J Curr Glaucoma Pract 2019;13:113-5. 